\title{
Artelogie
}

Recherche sur les arts, le patrimoine et la littérature de l'Amérique latine

$3 \mid 2012$

Image de la nation : art et nature au Chili

\section{Les « limbes » de Gerónimo de Vivar : représentations et appropriation de la nature chilienne pendant la Conquête}

Guillaume Gaudin

\section{(2) OpenEdition}

Édition électronique

URL : https://journals.openedition.org/artelogie/6762

DOI : 10.4000/artelogie.6762

ISSN : 2115-6395

Éditeur

Association ESCAL

Référence électronique

Guillaume Gaudin, «Les « limbes » de Gerónimo de Vivar : représentations et appropriation de la nature chilienne pendant la Conquête ", Artelogie [En ligne], 3 | 2012, mis en ligne le 12 septembre 2012, consulté le 07 janvier 2022. URL : http://journals.openedition.org/artelogie/6762 ; DOI : https:// doi.org/10.4000/artelogie.6762

Ce document a été généré automatiquement le 7 janvier 2022.

Association ESCAL 


\title{
Les « limbes » de Gerónimo de Vivar : représentations et appropriation de la nature chilienne pendant la Conquête
}

\author{
Guillaume Gaudin
}

«Se ofrece a los cavalleros españoles que quedays en anparo y sustentaçion d'esta ciudad de Santiago, la qual todos en general y cada vno en particular la debe tener por nueva patria» Pedro de Valdivia s'adressant à ses hommes en 1540 d'aprés Geronimo de Vivar (VIVAR [1558]

1979: 67)

1 La page de titre de la Coronica y rrelaçion copiosa y verdadera de los rreynos de Chile dévoile légèrement la vie de son auteur, Gerónimo de Vivar: un lieu, «natural de la çiudad de Burgos», et une date à laquelle l'œuvre est achevée, le 14 décembre 1558 (VIVAR, 1979 : 2). Le manuscrit est dédié au prince des Asturies, Charles (1545-1568) fils de Philippe II. La suite du texte ne nous en apprend guère plus. Assez pour affirmer que Vivar est un soldat partie prenante «dans ces provinces du Chili de sa découverte, conquête, peuplement et maintien avec don Pedro de Baldivia » (VIVAR, 1979:3). Il se trouve aux Indes depuis plusieurs années quand il rejoint Valdivia au Pérou pour entreprendre la conquête du Chili ${ }^{1}$.

2 Les années 1540-1550 constituent un tournant dans l'histoire du Nouveau Monde : l'ère des grandes conquêtes (Mexique, Pérou) se termine et la Couronne s'efforce de prendre le contrôle de la situation (définitivement acquis en 1573). Après l'échec de l'expédition d'Almagro (1525-1537) au Chili, les années 1540 sont marquées par les troubles au Pérou : les rivalités et la suppression de l'encomienda par la Couronne (1542) mettent le feu aux poudres. Pedro de Valdivia qui lutte dans le camp pizarriste se voit récompensé par la conquête du Chili qu'il commence en 1540 (il revient au Pérou en 1548 pour 
soutenir le vice-roi La Gasca contre Gonzalo Pizarro). Il fonde plusieurs villes mais la résistance des Indiens araucans ou mapuches empêche toute installation durable et paisible des colons. La dureté des combats entraine une escalade de terreur. Les Araucans se fédèrent, adaptent leur tactique militaire au nouvel ennemi et remportent victoire sur victoire. Valdivia est capturé et tué en 1553. Le nouveau gouverneur Francisco de Villagrán doit se replier et applique la tactique de la terre brûlée. Les rivalités entre les capitaines espagnols accentuent le chaos. En 1557, le vice-roi du Pérou, le marquis de Cañete nomme son propre fils comme gouverneur. La Crónica s'achève en 1558 alors que la guerre reprend de plus belle ${ }^{2}$. À partir de là, l'histoire du manuscrit de Vivar est mal connue.

3 Il est peut-être conservé au Conseil des Indes à Madrid. Au XVIIe siècle, Antonio de León Pinelo et Juan Díez de la Calle, deux officiers avides d'informations sur les Indes occidentales, y font référence dans leurs œuvres ${ }^{3}$. Longtemps perdu, le document réapparaît au XXe siècle; il est aujourd'hui conservé à la Newberry Library de Chicago et sa première publication ne date que de 1966. L'œuvre de Vivar, "premier chroniqueur chilien", offre plusieurs pistes de réflexion pour l'histoire des représentations de la nature chilienne.

\section{Mobilisation ibérique : une description au service de la Monarchie catholique}

4 Les 107 folios de la Crónica rapportent les heurs et malheurs de la Conquête du Chili. Comme tant d'acteurs de la Conquista, Vivar souhaite conserver la mémoire des hauts faits de don Pedro de Valdivia et de ses compagnons: «cosas dinas de perpetua memoria (...) y los casos aconteçidos que cada vn dia aconteçian vilos quedar en olvido (...)» (VIVAR: 3). Le récit prend d'abord la forme d'une biographie à la gloire de Valdivia pour se poursuivre jusqu'en 1558. Chronique de guerre exaltant les faits glorieux des sujets d'une Monarchie combattant sur tous les fronts, l'épopée de Valdivia commence dans les Flandres puis en Italie (Milan, Naples), se poursuit au Pérou pour terminer au Chili; Vivar n'oublie pas de rappeler que les ancêtres de l'Estrémègne combattirent les Maures. Par ailleurs, le royal dédicataire est qualifié à deux reprises de «más alto y mayor prinçipe del mundo»; dans le même paragraphe, «mundo» est répété quatre fois. Des deux côtés de l'Atlantique, l'Espagne triomphe : «los españoles, hijos de nuestra España (...) es tanta su valerosidad que en todos los negoçios que emprenden procuran subir a más y valer más» (VIVAR: 3) ; «españoles» et «nuestra España» sont martelés. Vivar affiche d'autant plus facilement sa fidélité à la Couronne après l'épisode de la rébellion de Pizarro.

5 À côté de cette entreprise de propagande impériale, Vivar, doté d'un indéniable sens pratique, se lance dans une description dont l'objectif est de recueillir un maximum de savoirs sur la nature et la population à conquérir : «contaré toda la conquista y las çiudades que se poblaren, las prouinçias que se descubrieren, $y$ tenples de tierra y de arboles y de yervas $y$ de rrios tan cavdalosos y de todos los puertos de mar que se descubrieren, y en los grados que cada vno está (...) y de las diferençias de lenguas y diferentes traxes y de sus costumbres y rritios $y$ çirimonias tan diferentes (...)» (VIVAR: 4). Il propose un véritable manuel à l'usage du colonisateur: "para que los que leyeren o oyeren esta rrelaçion se animen a semejantes descubrimientos, entradas y conquistas y poblaciones, y en ellas empleen sus animos y esfuerços en servicio de sus prinçipes y señores» (VIVAR: 3). En somme, la chronique offre un récit exaltant les exploits de Pedro de Valdivia et des Espagnols au Chili ainsi qu'une description précise des conditions naturelles et humaines pour la colonisation espagnole. L'ensemble, non exempt de références antiques (Ptolémée, Tite Live et 
Valère Maxime), s'affiche clairement au service de la Monarchie hispanique et de la foi catholique. Au croisement de diverses traditions littéraires (récit de voyage, historiographie antique, chroniques de la Reconquista et des croisades, oralité, description géographique), l'œuvre s'inscrit dans la «mobilisation ibérique » qui se développe au XVIe siècle à l'échelle planétaire depuis la Castille (Gruzinski, 2004 : 37-39). Savoirs, exaltation et faits d'armes concourent ensemble au rayonnement de la couronne, à l'appropriation de vastes territoires et à l'assujettissement de populations entières.

\section{Un approche sensible de la nature chilienne}

6 L'œuvre de Vivar et ses rapports à l'espace chilien appellent une analyse à l'aune des travaux d'Alain Corbin sur l'histoire des sensibilités. «Ose libremente andar por el mundo» déclare Vivar à son prince. La chronique s'inscrit dans la tradition des récits de voyageurs (marchands, guerriers, moines, etc.) qui rapportent, non sans exotisme, leurs aventures. Le «je» et l'aventure personnelle sont les moteurs et les garants légitimes du récit: «de lo que yo por mis ojos vi y por mis pies anduve y con la voluntad seguy» (VIVAR: 3). La volonté est forte de fixer et de préserver la vérité par l'observation directe (GONZÁLEZ SÁNCHEZ, 2007 : 33). Le récit de Vivar se prête donc bien à l'analyse du sensible : « le paysage est une lecture, indissociable de la personne qui contemple l'espace considéré. Évacuons donc ici la notion d'objectivité » (CORBIN, 2001: 11). En effet, la chronique n'est pas seulement une "histoire-batailles ", elle propose parallèlement une «vision cynégétique de la nature " ${ }^{4}$. Cette représentation fut une construction d'autant plus nécessaire dans le cadre d'un monde nouveau à conquérir. À ce titre, la Crónica de Vivar a pu servir de matrice pour l'image du Chili ${ }^{5}$.

\section{Une identité plurielle : esprit de possession, esprit créole, nation}

7 Il serait totalement anachronique de parler de nationalisme, tant espagnol, américain que chilien, pour le XVIe siècle. La construction identitaire dans les mondes hispaniques modernes, d'autant plus dans le domaine américain, s'avère plurielle et complexe. Bernard Lavallé a montré comment le passage du conquérant au créole s'opéra sans rupture, « être criollo, était plus lié à un état d'esprit, à une adhésion à des intérêts locaux, qu'à la naissance en terre américaine» (LAVALLE, 1982: 359). En témoignent les propos de Valdivia invitant à l'appropriation, en exergue de cet article. Or, l'« apparition de la conscience créole » repose notamment sur le rapport entretenu avec la nature américaine.

8 Comment le premier chroniqueur espagnol de la Conquête du Chili construit-il une représentation permettant aux Espagnols de s'approprier l'espace chilien? Quelles influences la nature et les habitants du Chili ont-ils sur le processus de construction identitaire des conquérants? Notre propos est de comprendre comment le premier chroniqueur espagnol de la Conquête du Chili construit un récit qui permet aux Espagnols de s'approprier l'espace chilien.

\section{Observer le Chili : un espace sauvage}

\section{Un paysage grandiose : fleuves, cordillères et océan}

9 Vivar offre la description d'une contrée inconnue, perdue à l'extrême sud du continent américain, accessible seulement après de longs mois de voyage. Il livre une image précise du paysage et de l'espace pour faire du Royaume du Chili une partie à part 
entière de l'Empire espagnol. Cette importance accordée au regard du chroniqueur, gage d'authenticité, est peut-être à mettre en lien avec un fait culturel plus général établi par les travaux d'Alfred Crosby : la supériorité, depuis la Renaissance, de la vue sur les autres sens comme médium de la rationalité (CROSBY, 1997).

Chez les chroniqueurs du XVIe siècle apparaissent les éléments récurrents de la nature chilienne : en premier lieu « la Cordillera Nevada qui se prolonge sur tout le Chili », puis les nombreux fleuves «de mucha agua ou caudaloso» et les lacs, les volcans, les longues pluies hivernales. Vivar décrit des fleuves profonds, furieux, larges, à fort débit, sablonneux (hondos, furiosos, anchos, caudalosos, arenosos) et rarement calmes (sesgos). Les rivières se transforment l'été en torrents qui entravent les communications: «quando allegó alla con sus españoles, con travajo a cavsa de los muchos y furiosos rrios que en aquella sazon avia...» (VIVAR: 110). Dans la vallée de Mapocho, la force des courants s'accroît avec les dénivellations et les rivières se transforment en éboulements : «En estas provinçias vienen los rrios cavdalosos y muy furiosos a cavsa de tener çerca la corrida (digo desde adonde naçe de las syerras nevadas fasta la mar) y por ser tan agra la baxada para correr. (...) y con la furia qu'el agua lleva rrodan las piedras y son muy peligrosos» (VIVAR: 87). La traversée du fleuve Bío-Bío emporte un commandeur espagnol et, en 1557, elle demande cinq jours au gouverneur Hurtado de Mendoza.

11 L'autre marqueur du paysage chilien est la Cordillère des Andes à laquelle Vivar consacre un chapitre entier, à la mesure de cet ensemble continental (Vivar: 163). Il ne manque pas de détailler son franchissement d'ouest en est. Les prétentions de Valdivia sur la province de Cuyo et les terres orientales sont en effet freinées par l'absence de col et les conditions extrêmes. La Cordillère forme un autre obstacle dans sa partie occidentale et littorale. Cependant, lorsqu'elle disparaît du paysage, les Espagnols sont désorientés comme lors d'une expédition au sud de Valdivia : «dio la buelta el gouernador syn ver más tierra, porque fuymos por entre la mar y la cordillera nevada por medio del conpas que ay de tierra, que no vimos la mar ni la cordillera nevada...» (VIVAR: 198)

12 À partir de Concepción, des forêts tempérées se dessinent de chaque côté de la plaine centrale. De Concepción à Valdivia les arbres sont variés, à l'image des grands conifères que certains Espagnols appellent libano car la résine a l'odeur de l'encens : " $Y$ estos arboles son rrobles y arrayanes $y$ de los avellanos que tengo dicho. Ay gran cantitad de cañas maçicas. Estos montes en alguna parte son rralos y en otras, muy espesos» (VIVAR: 188).

13 Le paysage du Chili est caractérisé par son climat extrême. À côté du tableau climatique régional, Vivar décrit la violence des éléments (VIVAR: 99). Il insiste également sur l'inversion des saisons dans l'hémisphère sud (VIVAR: 110).

14 Il évoque fréquemment l'océan: cordon ombilical avec le Pérou mais aussi espace propice à l'imaginaire. Lors de l'expédition qui doit certifier la découverte du détroit de Magellan, la côte prend des aspects fantastiques: «Aqui vimos vna cueva muy grande con vn pilar en medio hecho de la mesma peña, que cabrian en ella más de mill hombres, (...) Y pusymosle por nonbre la Cueva Ynfernal por su grandeza» (VIVAR: 213). L'aventure des grands espaces au prisme de l'imaginaire occidental amène des visions fantastiques.

\section{Les « cosas admirables » du Chili}

15 Au fil de la Crónica, le lecteur est tenu en haleine par l'enthousiasme du découvreur. Le narrateur met en scène son aventure personnelle et présente son goût, dans l'air du temps, pour les curiosités et les nouveautés. À l'instar de tant d'auteurs et homines 
viatores, Vivar souhaite révéler des faits inédits et avoir la primeur de ces mirabilia ${ }^{6}$. Bien qu'il s'agisse d'un passage obligé de toute chronique sur les Indes occidentales, Vivar doit justifier ses fantaisies: «Puesto que sea fuera de nuestro proposyto y derecho camino que llevamos, acordé, porque no quedasa en oluido, contar cosas admirables que ay en esta provinçia, las quales vi syguiendo esta jornada» (VIVAR: 15). Ces apartés sont pourtant fondamentaux car ils permettent d'atteindre un paroxysme dans le partage de l'expérience: «Es cosa admirable para quien lo pasa y ve, como para quien no lo ha visto y lo oye» (VIVAR: 25).

16 Aussi relève-t-on dans le manuscrit treize occurrences de "cosas admirables». Il existe une graduation ou une variation dans l'admirable depuis l'« étrange à voir » (extraño de ver) comme un arbre inconnu, l'«assez admirable» (harto admirable) à propos des victoires d'une poignée d'Espagnols contre une armée d'Indiens, les choses dont on peut «s'émerveiller" (maravillar) à propos de la fertilité de la terre, et enfin le summum (cosa milagrosa) lorsque Dieu fait surgir une source en plein désert.

Les chapitres 10 et 11 de la Crónica sont consacrés aux merveilles du désert d'Atacama qui impressionnent grandement Vivar : «La costelaçion d'esta provinçia o, por mejor dezir, desierto, es tan diuersa, qu'es cosa admirable (...) Ay en ésta más que dezir que de todas» (VIVAR: 25). D'abord, le chroniqueur exprime sa surprise de voir des cadavres momifiés huit ans après l'expédition de Diego de Almagro. Ensuite, réalisant les dangers de la traversée, Vivar donne les indications pour éviter de fâcheux désagréments aux "pelegrino conquistador»: "Los que pasan en tienpo de ynvierno, españoles $v$ yndios, que de frio $v$ de hanbre $v$ de sed mueren» (VIVAR: 26) ${ }^{7}$. L'extrême salinité de l'eau le laisse coi : «Es cosa admirable que en tanto que esta agua corre es clara, y tomada en vaso de plata $v$ de barro sacandola de su corriente, se quaja, y se hace tan blanca como el papel, luego en aquel momento que la sacan» (VIVAR: 25).

Le manque d'eau dans cette région est pour le chroniqueur un sujet intarissable qui transforme le voyage de l'ost en une quête héroïque. Lorsque le précieux liquide apparaît, il se prête à des jeux étranges que Vivar se targue d'expliquer de manière très rationnelle contrairement aux Indiens qui attribuent le phénomène au caractère de la rivière: "Allegaron a un rrio chico que corre poca agua, tanta que de vn salto se pasara. Comiença a correr a las nueve de la mañana, quando el sol calienta la nieve qu'está en vna rrehoya. Corre con grande furia, y haze mucho rruydo a cavsa del sytio donde corre. Dura el correr d'este rrio hasta ora de nona. Quando el sol baxa, haze sonbra vna alta syerra a la nieve qu'está en la rrehoya dicha, y como le falta el calor del sol, no se derrite la nieve, a cuya cavsa dexa de correr. Secase este rrio de tal manera y suerte que dizen los yndios-que mal lo entienden - que se buelue el agua arriba a la contra de como a corrido. Por tanto le llaman los yndios 'Anchallulla', que quiere dezir 'gran mentiroso'» (VIVAR: 24).

Dans cette nature hostile, les miracles ne sont pas exclus et de la main de Dieu naît une verte vallée, comme un encouragement à l'entreprise espagnole: "estava vn valle chico con poca agua clara y dulçe, que Dios fue servido de darla alli. Pareçe cosa milagrosa, porque no tiene sytio para manar ni venir de parte alguna» (VIVAR: 27).

Les indigènes rencontrés suscitent également l'étonnement de Vivar. Comme pour la nature, il éprouve un sentiment ambigu, entre admiration et dégoût du nouveau et de l'autre. C'est souvent à propos de leur engagement militaire que Vivar s'émerveille des Indiens: «los yndios que dentro de la fuerça estavan, y alçaron gran alarido, y dispararon sus flechas en tanta cantitad que hera cosa admirable» (VIVAR: 85). La construction des forts indiens (pucuran) est également digne d'enthousiasme: «Estava esto tan bien hecho como 
pueden los españoles hazer vna trinchera para defenderse de la artillera [...] La entrada tenian d'esta puerta los yndios çerrada con muy fuertes tablones gruesos, que hera cosa admirable.» (Vivar: 97) Les actes guerriers espagnols n'en sont pas moins admirables. D'abord, ce sont les faits d'armes du capitaine Valdivia dont l'élan, «tuant et blessant " autour de lui, «était chose admirable à voir ». Puis le mode de conquête dans son essence même est extraordinaire: «iCosa harto admirable que quatro mill honbres de guerra con grandes ardiles, y estando en su tierra, teme a treynta honbres de a cavallo!» (VIVAR: 90).

La " chose admirable " participe des stéréotypes sur les Indiens d'Amérique, à l'instar du cannibalisme : «E querido hazer minsion d'esto porqu'es cosa admirable, $y$ çierto no lo osara poner por memoria, sy d'ello no tuviera muchos testimonios. $Y$ de comerse vnos a otros no es de maravillar... en esto los tiene el demonio, nuestro aversario... Y a mi pareçer les deve de dezir el dia del juizio» (VIVAR, 226). Les Indiens vivent dans la nature la plus profonde, la moins civilisée : les monts et les forêts.

\section{«Los lobos monteses »: les Indiens, élément sauvage du paysage chilien}

Vivar relève la dénomination inca relative aux Indiens vivant entre le fleuve Maypo et le fleuve Maule: «Visto los yngas su manera de biuir los llamaron "pomaucaes", que quiere dezir "lobos monteses"» (VIVAR: 165). "Purun auka» signifie en quechua "ennemi rebelle ». La traduction « loup montagneux » n'est pas anodine : le loup n'est pas un animal aimé dans l'imagerie occidentale. Or, l'adjectif «monteses» est redondant : l'Inca Garcilaso de la Vega dans ses Comentarios Reales rapporte que dans la langue quechua "montagneux" est une manière voilée de dire "sauvage ". L'identification entre Indiens et montagne/forêt insiste sur la dangerosité du milieu naturel peuplé d'êtres dont l'humanité n'est pas clairement attestée. De plus, les Indiens ne vivaient pas en communauté, fondement même de la société policée hispanique (Herzog, 2007: 507-538): «los yndios de qualquiera parte de Yndias, puesto que sean lo más d'ellos animosos, que se ayan visto con españoles, en viendolos, los temen y cometen lo que qualquiera animal yndomito y siluestre comete: qu'es apartarse de la presençia de tal conpañia nunca d'ellos jamas vista, sobresaltando su coraçon, cometiendo la huyda, y enjendrando oydio y rrencor, como personas salvajes $y$ en fin naçidos y criados en pecado...» (VIVAR: 53).

Les Indiens sont rejetés dans le monde sauvage et démoniaque. Ils refusent de faire les semis et préfèrent mourir que de servir les Espagnols ${ }^{8}$. Vivar explique une telle attitude : «esto proçede de jente sylvestre, faltos de amor y caridad. $Y$ por ser tan avasallados del demonio, que los atrae a la muerte, antes que vengan al conoçimiento de la verdad por la amonestaçion de los christianos...» (VIVAR: 74). Par ailleurs, leur supériorité militaire s'explique par leur capacité à faire corps avec la nature : «Y visto por los yndios, alçaron tan gran bozeria, que pareçia que todo el mundo estaua alli, y que los montes se asolavan y talavan» (VIVAR: 85). La nature forme un rempart indestructible et un refuge inexpugnable (VIVAR : 42)9. 


\section{La nature chilienne domestiquée par les conquistadores}

La Crónica y Relación Copiosa y Verdadera de los Reinos de Chile se révèle être un véritable guide d'installation pour le colon. L'espace est ordonné en vallées décrites précisément afin d'évaluer leur capacité à être peuplées et civilisées - pobladas ${ }^{10}$. Cet inventaire précis et exhaustif n'est pas sans rappeler l'entreprise plusieurs fois répétée des "Relations géographiques", ces rapports que les autorités régionales et locales hispano-américaines fournirent en réponse aux questionnaires émis par le Conseil des Indes dès 1530. Les Relations les plus connues sont celles de 1573 et 1577 caractérisées par leur ampleur (Musset, 2003: 135-161). Dès 1533, une cédule royale demande à l'audience de Mexico de "tener entera noticia de las cosas de esa tierra y calidades» comprenant la superficie et les dimensions des territoires, leur nom, leur position, «las calidades y extrañezas», "qué población de gentes hay en ella de los naturales poniendo sus ritos $y$ costumbres (...) qué puertos y ríos (...) qué animales y aves (...).» (Solano, 1988: 4) Bien des éléments de réponse à ces enquêtes se trouvent sponte sua dans la Crónica de Vivar, dont nous proposons ici un classement (suivant nos catégories contemporaines):

- Géographie physique et climat : position géographique et disposition de la vallée, son relief, ses dimensions, hydrographie, volcans; pluie, température, saisons.

- Économie: productions agricoles, fertilité des sols, arbres et fruits comestibles, herbes (comestibles et médicinales), animaux dont gibiers, volatiles, mines de sel, d'or, d'argent, de cuivre.

- Société (tableau précis des populations indiennes): apparence physique, habitudes vestimentaires, type d'agriculture et habitudes alimentaires, religion, langue, institutions, sociabilité, parenté avec les tribus déjà rencontrées et influences inca, bellicisme, armes.

- Réseaux urbains: fondation de villes, des forts, ports comprenant les localisations géodésiques et distances entre les villes.

\section{Dans un premier temps : profiter des ressources locales}

Dans la Crónica de Vivar, la nature chilienne est passée au crible d'une analyse raisonnée employant des unités de mesure : le stade, la palme pour donner la hauteur d'une plante, les latitudes, etc. (Larraín Barros, 1990: 271-291). Aussi est-ce par comparaison avec une plante espagnole ou un objet connu qu'il transmet le mieux la représentation de ce qu'il voit: telle plante possède la tige du blé, les graines ressemblent à celles de moutarde, un cactus à la forme d'un cierge. La chronique de Vivar est aussi un recensement des ressources du Chili et de l'utilisation qu'en font les Indiens.

Dans le désert d'Atacama, la connaissance des maigres denrées alimentaires est une question de survie (VIVAR: 27). Ailleurs, Vivar décrit frijoles, maïs, papas et quinoa; cette dernière retient spécialement son attention: «...esta quinoa es vna yerba como bledos. Lleva unos granitos e vna espiga o dos o tres, que de en los cogollos que lleva. Es tan alta como un estado y menos. $Y$ los granitos que digo son a manera de mostaza y mayores. Quezen estos granitos los yndios e comenlos.» (Vivar: 37 ) Avec l'algarrobo et le maïs les Indiens font une chicha (VIVAR: 27). Un arbre, le "palo colorado», semblable au bois de Brésil, monopole royal, attire l'attention du chroniqueur. Dans la vallée d'Aconcagua, 
l'algarrobo est l'arbre de grande envergure par excellence, très utile pour la construction (VIVAR: 54 ).

C'est au sujet d'une ressource extrêmement importante dans les sociétés d'Ancien Régime, le sel, que Vivar ose la comparaison avec l'Espagne en faveur du Chili : «Esta sal d'este rrio es tan fina y tan blanca y dura y tan salada, que haze ventaja a todas las que yo $e$ visto, que son ynfinitas, asy en Peru como en Atacama, como en España, en salinas y en veneros, en piedras y en minas, y si acaso este rrio pasara por mitad de Castilla, quitara a Atiença y aun a otras partes.» (VIVAR: 25)

La description précise du climat, du régime des précipitations et de l'hydrographie a pour objectif d'évaluer les capacités agricoles des nouvelles terres à coloniser. Pour les hommes du XVIe siècle, l'agriculture, en particulier la céréaliculture, est une marque de civilisation, rempart contre la barbarie des Indiens nomades. Vivar se livre donc à l'observation des activités agricoles chez les populations indigènes (LARRAIN BARROS: 271). Cette expérience mène à un processus d'acculturation : «E querido dar quenta de las sementeras, y como se haze en esta provinçia syn travajo, qu'es Dios servido, darlo ansy, porque lo vi, y d'él me sustenté como los demas» (VIVAR: 54). Néanmoins, il laisse les produits autochtones à la consommation des Indiens : l'herbe qui ressemble au sarment est un «buen mantinimiento para ellos».

Le chroniqueur relève la présence de canaux d'irrigation - acequias - dans la vallée de Huasco et celle d'Aconcagua. Pour arroser les chácaras «tienen sacado los naturales XX y dos açequias grandes» (VIVAR: 50). Le regard est plus sévère concernant les Pormaucaes : «senbravan muy poco, y se sustentavan el más del tiempo de rrayzes de vna manera de cebollas» (Vivar: 165). Néanmoins, la région au sud du fleuve Itata offre de bonnes perspectives aux cultures hispaniques (Vivar : 182).

\section{S'enrichir (malgré tout) : encomiendas et métaux précieux}

Le mode de la Conquête réside dans l'institution coloniale par excellence : l'encomienda. Cette figure juridique qui date de 1503 désigne une communauté indienne soumise directement à un colon, conquistador encomendero. La répartition des encomiendas ne nécessite pas, du moins dans un premier temps, de connaissances précises des populations et territoires répartis entre les conquistadores. Cet aspect apparait clairement lors de la première entrée de Pedro de Valdivia dans le sud de l'Araucanie. À peine parvenu sur les rives du fleuve Cautín, l'Estrémègne procède à l'attribution des encomiendas sur un territoire non encore maitrisé. De même, au moment de la fondation de Santiago, «no tenía la claridad de todos los caçiques de toda la tierra... Y d'esta suerte rrepartio todos los caçiques y rrepartimientos, con sus yndios que a los tales caçiques eran sujetos, en sesenta pobladores» (VIVAR: 94). Guillaume Boccara a souligné que le repartimiento s'avérait inefficace et violent dans ces conditions particulières (BOCCARA, 1998 : 207). En ce sens, Gerónimo de Vivar voit juste dans le jeu de ses compagnons : «... a los conquistadores se les haze grave el senbrar y cultivar la tierra, prençipalmente aquellos que los dexaron en Castilla» (VIVAR: 74). À défaut de pouvoir offrir à ses soldats une encomienda, Pedro de Valdivia organise le territoire à la manière hispanique en mettant en place un domaine familial: «padre para los favorecer con lo que pude y dolerme de sus trabajos, ayudándoselos a pasar como de hijos, y amigo en conservar con ellos; zumétrico en trazar y poblar; alarife en hacer acequias y repartir aguas; abrador y gañán en las sementeras; mayoral y rabadán en hacer criar ganados....11 (Esteve Barba, 1960:10) Ces difficultés 
attestent la crise du modèle initial de colonisation par l'encomienda: les Lois nouvelles (1542) y ont mis un coup d'arrêt au profit du pouvoir royal. L'exploitation des Indiens prit d'autres formes et l'entreprise de Valdivia, calquée sur le modèle des Cortès et des Pizarro, s'avéra anachronique.

Le décompte de la main d'œuvre indigène est une priorité pour Vivar. Il est ainsi bien conscient du choc démographique de la Conquête : «No ay tanta gente es esta provincia [de Mapocho] como quando los christianos entraron en ella, a cavsa de las guerras y alçamientos que con los españoles tuvieron, fue parte para desmenuyllos, que tres partes, no ay la una. Y las minas an sido tanbien parte, que lo vno con lo otro se a juntado el destruymiento d'ellos.» (VIVAR: 161)

Avec l'encomienda, les métaux précieux sont l'autre moteur de la Conquête: «a las entradas en estas tierras nuevas, como no aya semejante metal van los españoles de mala gana (...). Y como es metal tan codiçioso, y que por él vienen los españoles a estas partes más que por otra cosa, segun pareçio fue cavtela para dividir los christianos.» (VIVAR: 61) Vivar affirme que l'or doit «acreditar este rreyno» auprès des autorités péruviennes et péninsulaires. Aussi la Crónica recense-t-elle les mines d'or et d'argent, de cuivre, de sel et matières nobles, comme dans l'Atacama: "Ay mucho alabastro. Ay ansy mismo muchas y muy infinitas colores : colorado y azul, dacle vltramarino, que alla se nombra en Castilla...» (VIVAR : 20).

Vers Santiago une première mine est exploitée par les Indiens du cacique Michimalongo. Valdivia s'emploie rapidement à l'organiser : l'or, la main d'œuvre, une sortie maritime, du bois. Mais les Indiens ne se laissent pas exploiter si facilement et tuent les colons. À travers ce récit, on saisit l'organisation de la mine: six cents travailleurs indiens (yanaconas et indigènes) et des esclaves noirs (VIVAR: 62 - 63) surveillés par quinze Espagnols. Dans la région de Valdivia, de nouvelles mines sont découvertes et une villa au nom prometteur est fondée : Villarica (VIVAR: 188).

Dès lors, les mines deviennent des enjeux stratégiques et sont la cible des Indiens insurgés avec à leur tête le fameux Lautaro, plus tard glorifié par Alonso de Ercilla et Pablo Neruda: «Los vezinos de la çiudad de Santiago tienen las minas junto al rrio Mavle, $y$ estavan syete españoles en ella. (...) Avisados los españoles que en las minas estavan que venía Lavtaro, desanpararon las minas» (VIVAR: 223).

\section{Identifier l'espace}

\section{Les choses de « nuestra España »}

Les références hispaniques, courantes dans le texte de Vivar, font partie des critères d'évaluation de la qualité et de la productivité d'une vallée. À propos de la région de la Concepción: «Y se daran todas las demas plantas de nuestra España muy bien, (...) ay oroçuz que produze la tierra, que en Castilla la Vieja llaman rregalizia (...)» (VIVAR: 182). Au chapitre 140, Vivar dresse l'inventaire de toutes les plantes hispaniques qui poussent aussi bien voire mieux que dans la Péninsule ibérique : «E todo esto se da tan bien como en la parte que mejor se da de nuestra España. E se daratodo lo demas que se truxere» (VIVAR: 252). En effet, tout est " très bon ", les melons, les choux, «ay viñas, y en ninguna parte de Yndias se a dado tan buena vua», "las demas arboles que se traygan de nuestra España se dara muy bien.» Les premiers à tenter l'expérience sont cités en héros: «el primer honbre que lo hizo en esta tierra fue vn vezino que se dize Rodrigo de Araya.», le premier éleveur de cheval 
est «Rodrigo Gonçales, natural de Carmona». Vivar entrevoit le potentiel de la fertilité du Chili : «hazese ya tanto vino que basta para esta gobernaçion, y que pueden prover a otras partes.» Tout ceci montre la volonté des conquistadores: reproduire une nouvelle Espagne au Chili, si possible meilleure encore, où l'Espagnol reste oisif. On trouve donc chez Vivar les racines de l'esprit colon, cette volonté d'être chez soi dirigée d'abord contre les Indiens (LAVALLE, 1982: 356). Or, l'image d'un territoire domestiqué tient difficilement face à la réalité d'une conquête inachevée.

\section{Le Chili : un territoire hostile, fantastique, donc héroïque}

La première rencontre de Vivar dans le désert d'Atacama est funèbre : les cadavres de l'expédition d'Almagro. Un trait de la Conquista s'exprime de manière extrême au Chili : la «lutte de poignées d'hommes affamés et brisés de fatigue, contre des multitudes » (Chaunu, 1971: 21). Contrairement aux conquêtes précédentes, celle du Chili ne se fit pas en un éclair : au moment où Vivar achève sa Crónica, les guerres chiliennes sont loin d'être finies. Il témoigne très bien de cet état d'esprit et de la peur qui saisit les Espagnols : aux alentours de Santiago, «...los españoles, syendo tan pocos en cantitad, y tan pelegrinos y apartados de donde socoro les viniese, acometer a tanta barbarica gente y tan guerreros...» (VIVAR: 102).

37 En effet, le chapitre 60 rapporte le retour d'un lieutenant de Pedro de Valdivia, Alonso de Monroy, parti chercher des secours au Pérou. Les retrouvailles ont lieu le 1er janvier 1545, après deux ans de séparation. Vivar les décrit de cette manière : «Y el preguntar por las cosas de alla, y ellos por las de aca: preguntavan los de aca como honbres qu'estavan en el lynbo a los otros como a personas que venian del mundo. Demandavan los rrezien venidos lo que demandaron los del purgatorio a Dante Aligero, quando alla anduvo con la ynmaginaçion [...]» (Vivar: 109). La mention du lynbo et du purgatorio invite le lecteur à tracer un parallèle entre les mondes imaginaires, assimilés à l'au-delà, et les lieux géographiques. Ainsi, on peut établir les correspondances suivantes : le Chili coïncide avec les limbes, en bordure de l'enfer; le Pérou est le monde des vivants; le chemin séparant le Chili du Pérou (désert d'Atacama/ Cordillère) s'apparente au purgatoire. Faut-il voir dans les limbes de Vivar le lieu défini par la "géographie de l'au-delà " chrétien (LE GOFF, 1999: 775-1230) ? Les limbes sont destinés aux enfants morts sans avoir reçu le sacrement du baptême et aux justes de l'Ancien Testament ramenés des Enfers par le Christ. Chez Dante, le purgatoire est une montagne divisée en sept corniches étagés représentant les sept pêchés capitaux; les limbes sont la preuve de l'affection de Dante pour les auteurs païens et sa pitié pour les petits enfants morts en bas âge. De son côté Vivar rappelle à plusieurs reprises les pêchés des conquistadores comme l'orgueil, la paresse ou l'avarice. Dès lors, le purgatoire de l'Atacama et de la Cordillère serait le prix à payer pour racheter les pêchés des Espagnols et sortir des limbes, ce « lieu là-bas attristé (...)/ par des ténèbres, où les lamentations/ Ne sonnent pas comme cris, mais ne sont que soupirs » (Divine Comédie, VII, 28-33).

Si l'on ajoute que le latin limbus a le sens de bordure, on en déduit que Vivar choisit les images de la marge pour construire sa représentation du Chili. La toponymie des villes fondées par Valdivia conforte cette idée: Santiago del Nuevo Estremo fondée le 12 février 1541 et la ville de Los Confines. De plus, Vivar précise dans le chapitre 59, que les conquistadores «estavan tan desnudos quanto nunca lo estuvo gente en estas partes, ni en ninguna de Yndias». Or cette situation d'isolement est vécue comme un "âge d'or » en 
aparté de la Légende noire, et ne fait que rehausser les vertus morales et chevaleresques des conquistadores: «Digo bueno syn codiçia, sano syn maliçia y libre de avariçia. Todos hermanos, todos conpañeros, todos contentos (...) Llamavale yo a este tiempo "tiempo dorado"» (Vivar: 107). Le temps d'une conquête idéalisée est révolu : une sorte de nostalgie du vétéran commence à poindre dans l'esprit de l'auteur. L'œuvre de Dante offre comme celle de Vivar de nombreuses descriptions de paysages et d'épreuves successifs : ils ont en commun une représentation cinétique de l'espace où réel et imaginaire se mêlent.

\section{Le Chili : un territoire à conquérir}

Dans cet univers fantastique, l'appropriation peut passer par la construction d'une nouvelle identité reposant principalement pour le Chili sur l'existence d'un ennemi commun. Ce processus rejoint "la fonction "nationalisatrice" de la monarchie [hispanique qui] s'exerçait surtout à travers les guerres » (ALVAREZ JUNCO : 2011, 40). Dans les premiers temps de la Conquête, les Espagnols s'efforcent ainsi de développer un «diagramme souverain " (BOCCARA, 1998: 206). Il s'agit de la mise en place des institutions coloniales comme l'encomienda, puis d'un réseau de forts et de villes. Une des premières actions espagnoles réside dans le formalisme et la prise de possession symbolique de l'espace selon des rites bien précis. Ainsi, en arrivant au Chili, Pedro de Valdivia ne manque pas de célébrer la cérémonie de prise de possession. Une mise en scène complexe démultiplie les dimensions de la cérémonie : Valdivia clame la prise de possession devant son ost, des branches sont coupées, etc. L'écrit joue également un rôle primordial en apportant la preuve juridique de la prise de possession (VIVAR: 29).

La toponymie joue également un rôle important. Le nom de la terre conquise ne peut rester inchangé car il porte trop de significations négatives : «Dezianle los yndios a don Diego de Almagro que hazía en esta valle 'anda Chire', que quiere dezir 'gran frio'. Quedóle al valle el nonbre de Chire, corrompido el bocablo le llaman Chile. Y d'este apellido tomó la governaçion y rreyno el nonbre que oy tiene, que se dize Chile» (VIVAR: 50). En effet, en plus du sens négatif du mot, le Chili évoque également l'échec d'Almagro. Pedro de Valdivia voulut effacer cette réputation en donnant un nouveau nom : ce fut celui de sa province natale et celle de Pizarro, la Nouvelle Estrémadure.

41 Les rituels de rencontre avec les Indiens poursuivent le même but. Pedro de Valdivia fait appeler les caciques pour leur apprendre la venue des Espagnols et prononcer une forme simplifiée du requerimiento (VIVAR: 30). Selon Guillaume Boccara, "c'est à travers la lecture du requerimiento et par la magie du rituel qui lui était associée que les Espagnols prenaient possession d'un territoire et transformaient ses habitants en vassaux du roi » (BOCCARA, $1998:$ 209). Le caractère religieux est bien sûr primordial dans la Conquête: l'église est par exemple un moyen d'imposer dans le paysage la présence espagnole. Valdivia se lance alors dans une pastorale où l'esprit de domination est très appuyé : «Lo primero que hizo fue vna yglesya en que se dezia misa. (...) Dioles más a entender que a quien le syrve y guarda y cunple sus mandamientos [de Dios] le da la vida eterna, rreçibiendo agua de bautismo, y el que no creyer, ni rreçibiere nin cunpliere sus mandamientos rreçibiera pena perdurable syn fin» (VIVAR: 52).

Tous ces gestes jouent un double rôle. D'une part, il s'agit de mettre en confiance les Espagnols, de s'approprier le nouveau territoire et de placer ce dernier sous la protection de Dieu ou d'un saint (Jacques, la Vierge). La conquête est aussi 
évangélisatrice et le paysage doit être christianisé par l'église et son clocher. D'autre part, il s'agit de démonstrations de puissance que les Indiens doivent voir et assimiler. La confusion est volontairement maintenue entre le roi d'Espagne - «el gran apo de Castilla »-, Dieu et Valdivia. Nous ne traiterons pas ici de la fondation des villes, sujet largement traité par Vivar et processus bien connu de l'appropriation de l'espace par les Espagnols : « la ville, c'est l'Espagne en Amérique », insiste Thomas Calvo (CALVO, 1994: 127). Concentrons-nous sur des phénomènes plus spécifiques au Chili.

Lorsque la conquête était déjà bien avancée et que la résistance indienne s'accentua, les actes symboliques ne contentaient plus les exigences espagnoles. Le territoire serait marqué par leur présence et les Indiens devraient le ressentir jusque dans leur chair. Ce type d'exercice du pouvoir s'exprime dans les expéditions annuelles en territoire ennemi, véritables razzias. Ces campagnes estivales empruntent une sorte de parcours punitif à travers les localités rebelles. Vivar témoigne de cette pratique pour la région de Coquimbo où Valdivia a fait construire un fort sur le site de La Serena : «Cada dia corrian el valle los christianos, y matavana los que se defendian, y prendian a los demas, a los quales enbiavan por mensajeros a la gente de guerra. D'esta suerte se les hazía la guerra, aviendo los yndios rronpido la paz» (Vivar: 91) La violence atteint des sommets comme dans la vallée d'Aconcagua: "Luego fue venido el caçique Tanjalongo y presentado ant'el general, manóle cortar los pies por la mitad (...) Este es vn genero de castigo que para los yndios es el más conveniente y no matarlos, porque los biuos olvidan a los muertos, como en todas partes se vsa, $y$ quedando vno biuo y d'esta suerte castigado, todos los que lo ven se le rrepresenta el delito que cometio aquél, pues anda castigado. Y él propio lo tiene tan en memeria que avnque quiera, no puede olvidarlo» (VIVAR, 92). Le second gouverneur, Garcia Hurtado de Mendoza, fait usage du même genre de représailles que Valdivia, il trouve même de nouveaux supplices plus cruels, comme celui de couper les mains et le nez de cent cinquante captifs (VIVAR: 240).

La guerre contre les Indiens est extrêmement cruelle à l'image de cette scène où la nature offre le cadre grandiose - une lagune de six lieues - à la violence meurtrière espagnole: «Y no pudiendo rresistir la fuerça de los christianos, echaronse a nado, pensando escapar por alli las uidas. (...) Y como yvan cansados, se ahogaron todos, que fue vna lastima muy grande de ver tantos cuerpos sobreaguados y echados por aquella playa de la laguna» (VIVAR: 224).

Le résultat est contreproductif. Dans une scène fameuse, reprise dans La Araucana, Vivar décrit comment un Indien aux mains coupées retourne parmi ses frères : “"Estos con quien vays a pelear me los cortaron, $y$ lo mesmo haran a los que de vosotros tomaren, y nadie permita huyr syno morir, pues moris defendiendo vuestra patria." [...] él moriria primero, y que ya que no tenia manos, que con los dientes haria lo que pudiese» (VIVAR: 242). L'usage du mot "patrie" montre le passage d'une guerre de conquête vers un conflit durable entre deux nations. Valdivia est capturé à Tucapel et exécuté, d'après Vivar, par le chef Caupolicán d'un coup de lance. Le vainqueur est Lautaro, son ancien palefrenier, appelé par les chroniqueurs Mariño de Lobera et Bartolomé Escobar libertador, terme étonnant pour le XVIe siècle repris par les patriotes chiliens de l'indépendance ${ }^{12}$ - Vivar lui qualifie Lautaro de «mal yndio» (VIVAR: 202). 


\title{
Conclusion
}

\author{
«Pues hagamos Reyno a Chile» \\ Charles Quint, d'après Diego de Rosales ${ }^{13}$
}

la nature tient une place exceptionnelle (GERBI, 1978). Du fait de sa conquête particulièrement difficile, de l'hostilité de la résistance des peuples autochtones et d'éléments naturels particulièrement extraordinaires, le chili tient une place particulière. Il a d'emblée été présenté comme un espace hostile où les Espagnols, donc la civilisation, étaient mis en échec. Des marqueurs géographiques et topographiques sont rapidement mis en place : l'Atacama, la Cordillère des Andes, le fleuve Bío-Bío, les volcans, etc. Les Indiens rebelles sont délibérément associés aux aspects les plus sauvages de la nature : les forêts et les montagnes sont le siège de forces maléfiques qu'il faut combattre. On retrouve ici les thèmes des récits de chevalerie, voire de la croisade, qui légitiment l'usage massif de la violence. processus d'appropriation. Porteur à la fois de l'imaginaire médiéval et de la méthode humaniste, Gerónimo de Vivar est le premier Européen à décrire de manière précise l'espace chilien. Bien que son œuvre soit restée manuscrite jusqu'au XXe siècle, elle n'en est pas moins révélatrice et fondatrice de la représentation européenne et, en un sens, hispano-américaine, du Chili. Loin d'une vision romantique de l'espace, Vivar fournit les connaissances nécessaires à la colonisation. On mesure ici la capacité de la Couronne à réunir de telles informations sur les territoires fraîchement conquis rappelons que la chronique de Vivar circula vraisemblablement au Conseil des Indes. Spontanément le conquistador Vivar est capable de décrire précisément l'espace chilien. Il pense et organise le territoire en vallées potentiellement habitables et recense les ressources exploitables pour les futurs colons. Cette description « utilitariste » du Chili s'apparente volontiers à un prospectus appelant à l'émigration et à la colonisation.

Dans le même temps, il livre une représentation sensible et même symbolique de la nature: purgatoire, limbes et cavernes infernales sont convoqués pour faire comprendre les affres de la conquête. Le sentiment d'isolement de cette poignée d'hommes perdus dans les immensités, livrés aux «lobos monteses», marque durablement le rapport du colon au territoire. Il n'est dès lors pas exclu de trouver chez Vivar un discours exaltant la nature chilienne dans le but de créer une nouvelle patria chica " adoptive " : à la « sauvagerie » des Indiens rebelles intimement liés à la nature, Vivar oppose un paysage domestiqué, ordonné par la parole, les gestes, les villes mais aussi la violence hispanique.

L'adhésion au projet impérial n'est donc pas contradictoire avec une forme d'identité intimement liée à l'attachement des Espagnols à la communauté locale et au désir d'un «chez-soi » (HERZOG, 2006). On mesure le rapide processus d'appropriation, voire de créolisation, à l'aune des récits du XVIIe siècle comme ceux d'Alonso de Ovalle ou de Diego de Rosales. Nés au Chili, sujets du Roi Catholique, ils placeront la nature chilienne au cœur d'une identité revendiquée. 


\section{BIBLIOGRAPHIE}

ÁLVAREZ JUNCO, J. (2011), L'idée d'Espagne. La difficile construction d'une identité collective au XIXe siècle. Rennes, PUR.

ANTEI, G. (1989), La invención del Reino de Chile. Gerónimo de Vivar y los primeros cronistas chilenos. Bogotá, Instituto Caro y Cuervo.

BERNARD, C. et GRUZINSKI, S. (1993), Histoire du Nouveau monde. Les métissages, t. 2. Paris, Fayard.

BOCCARA, G. (1998), Guerre et ethnogenèse mapuche dans le Chili colonial, L'invention du soi. Paris, L'Harmattan.

CALVO, T. (1994), L’Amérique ibérique de 1570 à 1910. Paris, Nathan Université.

CARNEIRO ARAUJO, S. (2011), « La Crónica de Jerónimo de Vivar y la conquista de Chile ». In: Compostella Aurea. Actas del VIII Congreso de la AISO, 3 vols. Santiago de Compostela (487-493).

CASANOVA, W. (1991), « Réalité et exaltation de la nature "chilienne" dans la Crónica de Gerónimo de Vivar et dans l'Histórica relación d'Alonso de Ovalle ", In: La nature américaine en débat : identités, représentations, idéologies, Presse universitaire de Bordeaux (9-36).

CORBIN, A. (2001), L’homme dans le paysage. Paris, Broché.

CROSBY, Al. (1997), The measure of Reality. Quantification and western Society. 1250-1600. Cambridge University Press.

ESTEVE BARBA, F. (éd.) (1960), Crónicas del Reino de Chile. Ediciones Atlas, Madrid.

GAUDIN, G. (2011), « L’Empire de papiers de Juan Díez de la Calle, commis du Conseil des Indes. Espace, administration et représentations du Nouveau Monde au XVIIe siècle ». In : Nuevo Mundo Mundos Nuevos, Aula virtual. http://nuevomundo.revues.org/60322. Consulté le 27 juin 2012.

GAUDIN, G. (2006), « Gerónimo de Vivar y Juan Díez de la Calle : Dos representaciones del espacio iberoamericano en la época moderna ». In: Takwá, n. 9, Guadalajara. http://148.202.18.157/sitios/ publicacionesite/pperiod/takwa/Takwa9/geronimo_bibar.pdf

GERBI, A. (1978), La naturaleza de las Indias nuevas. México, FCE.

GONZÁLEZ SÁNCHEZ, C. A. (2007), Homo viator, homo scribens. Cultura gráfica, información y gobierno en la expansión atlántica (siglos XV-XVII). Madrid, Marcial Pons.

GREENBLAT, S. (1996), Ces merveilleuses possessions. Découverte et appropriation du Nouveau Monde au XVIe siècle. Paris, Les Belles Lettres.

GRUZINSKI, S. (2004), Les Quatre parties du monde. Histoire d'une mondialisation. Paris, La Martinière.

HERZOG T. (2007), « Terres et déserts, société et sauvagerie ». Annales. Histoire, Sciences Sociales n. 3. Paris, 62e année. (507-538).

HERZOG T. (2006), Vecinos y extranjeros. Hacerse español en la edad moderna. Madrid, Alianza.

LARRAÍN BARROS, H. (1990), “El paisaje fitogeográfico del Norte Chico y zona central chilenos y su utilización por el hombre en el siglo XVI. Visión de los cronistas tempranos". Santiago, Revista Chilena de Historia y Geografía, n. 158.

LAVALLE, B. (1982), Recherches sur l'apparition de la conscience créole dans la vice-royauté du Pérou. L'antagonisme hispano-créole dans les ordres religieux (XVI-XVIIe siècles), t. 1. Atelier national de reproduction des thèses-Université de Lille III. 
LE GOFF, J. (1999), Un autre Moyen Âge. Paris, Gallimard.

MUSSET, A. (2003), « Décrire pour gouverner. Les « Relations qui doivent être faites pour la description des Indes » de 1577 », In: Giorgio Bruno et Jean-Pierre Olivier de Sardan (dirs.), Pratiques de la description. Paris, EHESS.

SOLANO, F. de (éd.) (1988), Cuestionarios para la formación de las Relaciones geográficas de Indias. Siglos XVI/XIX. Madrid, CSIC.

VIVAR, G. de (1979), Crónica y relación copiosa y verdadera de los Reinos de Chile (1558). Berlin, Colloquium Verlag. Edición de Leopoldo Saez-Godoy.

VIVAR G. de (1966), Crónica y relación copiosa y verdadera de los reinos de Chile, Santiago, Fondo Histórico y Bibliográfico José Toribio Medina, Biblioteca Nacional de Chile. Disponible sur : http://www.memoriachilena.cl/temas/documento_detalle.asp?id=MC0008847

ZÚÑIGA, J. P. (2002), Espagnols d'outre-mer. Émigration, métissage et reproduction sociale à Santiago-duChili au 17e siècle. Paris, Éditions de l'EHESS.

\section{NOTES}

1. Sa véritable identité fait débat. Cf. ANTEI, 1989 et CARNEIRO ARAUJO, 2011.

2. Cf. Bernand et Gruzinski (1993), particulièrement, entre les pages 465 et 471.

3. Cf. Gaudin, 2012.

4. Cf. CASANOVA, $1991:$ 9-36 ; voir aussi, GAUDIN, $2006: 31-51$.

5. La Historia del descubrimiento y conquista del Perú (Anvers, 1555) de Agustín de Zárate puis La Auracana d'Alonso de Ercilla (1569) ont également joué un rôle important dans la «construction d'une légende » du Chili. Cf. Zuñiga, $2002:$ 55-71.

6. Le goût des mirabilia est typiquement médiéval, cf. LE GOFF, 1999 : 455-476. GREENBLAT, 1996, et GONZÁLEZ SÁNCHEZ, 2007 : 47-77.

7. Voir aussi page 28 pour une description précise du climat désertique.

8. Topos des récits de croisade : les infidèles, les juifs, préfèrent mourir que de se laisser convertir. Remarque orale d'Axelle Neyrinck.

9. Voir aussi les pages 95, 97 et 168 .

10. Pour le sens "civilisateur" du mot "poblado" cf. HERZOG, 2007.

11. Lettre de Pedro de Valdivia à l'Empereur, 4 septembre 1545, apud. ESTEVE BARBA,1960:10.

12. Bernand et Gruzinski, $1995: 468$.

13. ROSALES Diego de, Historia general del Reino de Chile, Flandes Indiano, 1674, apud ANTEI, 1989 : 24.

\section{RÉSUMÉS}

La chronique du conquistador Gerónimo de Vivar achevée en 1558 est à la fois un récit de la Conquête à la gloire du chef Pedro de Valdivia et une description précise de la nature, des paysages et des populations indigènes. Le poids des représentations et la volonté d'appropriation $\mathrm{du}$ territoire par le discours produisent un tableau de la nature chilienne au service des 
Espagnols. Par ailleurs, le royaume du Chili est progressivement caractérisé, identifié à sa nature et simultanément intégré à l'univers politique et culturel hispanique. Sans parler de créolisme, encore moins de nationalisme, on trouve dans la première chronique chilienne le souci d'inventer un « chez-soi », y compris par la brutalité contre les Indiens.

La crónica del conquistador Gerónimo de Vivar acabada en el año 1558 es a la vez una narración de la conquista glorificando el caudillo Pedro de Valdivia y una descripción precisa de la naturaleza, los paisajes y los pueblos indígenas. La importancia de las representaciones y la voluntad de apropiación territorial por parte de los discursos producen un cuadro de la naturaleza chilena al servicio del español. Por otra parte, el reino de Chile se va caracterizando gradualmente, en identificación con la naturaleza y al mismo tiempo integrado al mundo político y cultural hispánico. Sin hablar de criollismo, o tampoco de nacionalismo, encontramos en la primera crónica de Chile la voluntad de inventar un 'chez soi', incluso utilizando la brutalidad contra los indios.

\section{INDEX}

Mots-clés : Chroniques d'Indes, Gerónimo de Vivar, royaume du Chili, territoire, nature

Palabras claves : Crónicas de Indias, Gerónimo de Vivar, Reyno de Chile, territorio, naturaleza

\section{AUTEUR}

\section{GUILLAUME GAUDIN}

Université de Toulouse, FRAMESPA-UMR 5136. Maître de conférences en histoire moderne des mondes hispaniques.

guillaume.gaudin[at]univ-tlse2.fr 